\title{
Bilingual Text, Matching using Bilingual Dictionary and Statistics
}

\author{
Takehito Utsuro ${ }^{\dagger} \quad$ Hiroshi Ikeda $a^{\ddagger}$ Masaya Yamane Yuji Matsumoto $^{\dagger} \quad$ Makoto Nagao $^{\ddagger}$ \\ ${ }^{\dagger}$ Graduate School of Information Science \\ Nara Institute of Science and Technology \\ ${ }^{\ddagger}$ Dept. of Electrical Engineering \\ Kyoto University
}

\begin{abstract}
This paper describes a unified framework for bilingual text matching by combining existing hand-written bilingual dictionaries and statistical techniques. The process of bilingual text matching consists of two major steps: sentence alignment and structural matching of bilingual sentences. Statistical techniques are applied to estimate word correspondences not included in bilingual dictionaries. Estimated word correspondences are useful for improving both sentence alignment and structural matching.
\end{abstract}

\section{Introduction}

Bilingual (or parallel) texts are useful as resources of linguistic knowledge as well as in applications such as machine translation.

One of the major approaches to analyzing bilingual texts is the statistical approach. The statistical approach involves the following: alignment of bilingual texts at the sentence level using statistical techniques (e.g. Brown, Lai and Mercer (1991), Gale and Church (1993), Chen (1993), and Kay and Röscheisen (1993)), statistical machine translation models (e.g. Brown, Cocke, Pietra, Pietra et al. (1990)), finding character-level / word-level / phrase-level correspondences from bilingual texts (e.g. Gale and Church (1991), Church (1993), and Kupiec (1993)), and word sense disambiguation for M'T (e.g. Dagan, Itai and Schwall (1991)). In general, the statistical approach does not use existing hand-written bilingual dictionaries, and depends solely upon statistics. For example, sentence alignment of bilingual texts are performed just by measuring sentence lengths in words or in characters (Brown et al., 1991; Gale and Church, 1993), or by statistically estimating word level correspondences (Chen, 1993; Kay and Röscheisen, 1993).

The statistical approach analyzes unstructured sentences in bilingual texts, and it is claimed that the results are useful enough in real applications such as machine translation and word sense disambiguation. However, structured bilingual sentences are undoubtedly more informative and important for future natural language researches. Structured bilingual or multilingual corpora serve as richer sources for extracting linguistic knowledge (Klavans and 'Tzoukermann, 1990; Sadler and Vendelmans, 1990; Kaji, Kida and Morimoto, 1992; Utsuro, Matsumoto and Nagao, 1992; Matsumoto, Ishimoto and Utsuro, 1993; Ut- suro, Matsumoto and Nagao, 1993). Compared with the statistical approach, those works are quite different in that they use word correspondence information available in hand-written bilingual dictionaries and try to extract structured linguistic knowledge such as structured translation patterns and case frames of verbs. For example, in Matsumoto et al. (1993), we proposed a method for finding structural matching of parallel sentences, making use of word level similarities calculated from a bilingual dictionary and a thesaurus. Then, those structurally matched paralle] sentences are used as a source for acquiring lexical knowledge such as verbal case frames (Utsuro et al., 1992; Utsuro et al., 1993).

With the aim of acquiring those structured linguistic knowledge, this paper describes a unified framework for bilingual text matching by combining existing hand-written bilingual dictionaries and statistical techniques. The process of bilingual text matching consists of two major steps: sentence alignment and structural matching of bilingual sentences. In those two steps, we use word correspondence information, which is available in hand-written bilingual dictionaries, or not included in bilingual dictionaries but estimated with statistical techniques.

The reasons why we take the approach of combining bilingual dictionaries and statistics are as follows: Statistical techniques are limited since 1) they require bilingual texts to be long enough for extracting useful statistics, while we need to acquire structured linguistic knowledge even from bilingual texts of about 100 sentences, 2) even with bilingual texts long enough for statistical techniques, useful statistics can not be extracted for low frequency words. For the reasons 1) and 2), the use of bilingual dictionaries is inevitable in our application. On the other hand, existing hand-written bilingual dictionaries are limited in that available dictionaries are only for daily words and usually domain specific on-line bilingual dictionaries are not available. Thus, statistical techniques are also inevitable for extracting domain specific word correspondence information not included in existing bilingual dictionaries.

At present, we are at the starting point of combining existing bilingual dictionaries and statistical techniques. 'Therefore, as statistical techniques for estimating word correspondences not included in bilingual dictionaries, we decided to adopt techniques as simple as possible, rather than techniques based-on complex probabilistic translation models such as in 


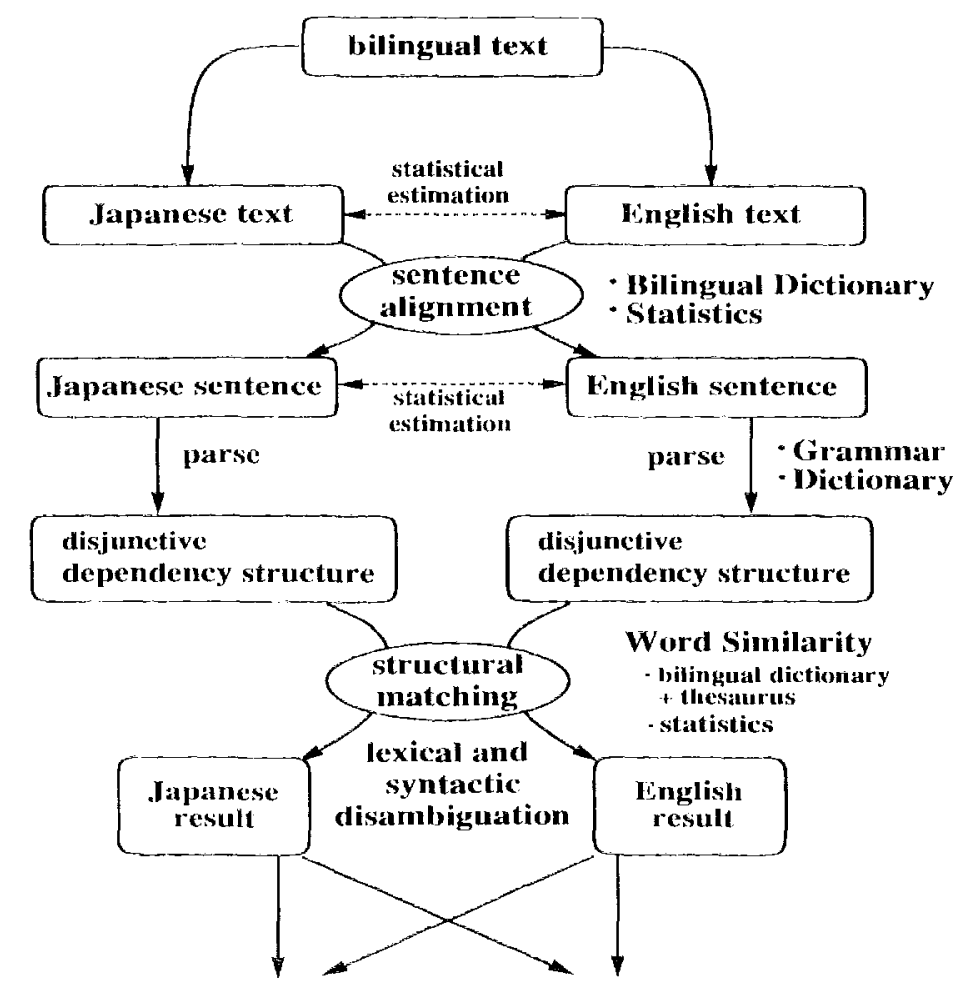

Lexical Knowledge, Translation Patterns

Fig. L: 'The Franework of Bilingual 'lext Matehing

Browu et al. (1990), Brown, Pietra, Pietra and Mercer (1993), and Chen (1993). What we adopt are simple co-occurrence-frequency-based techniques in Gale and Church (1991) and Kay and Röscheisen (1993). $\Lambda$ s techniques for sentence alignment, we adopt also quite a simple nethod based on the number of word correspondences, without any probabilistic translation models.

In the following sections, we illustrate the specifications of our bilingual text matching framework.

\section{The Framework of Bilingual Text Matching}

The overall framework of bilingual text matching is depicted in Fig. 1. Although our framework is inplemented for Japanese and Finglish, it is language independent.

First, bilingual texts are aligned at sentence level using word correspondence information which is a vailable in bilingual dictionaries or estimated by statis tical techniques. "Statistical estimation" at text level indicates that length-based statistical techniques are applied if necessary. (At present, they are not implemented.) "Statistical estimation" at sentence level indicates that word-to-word correspondences are estimated by statistical techniques. Then, each mono- lingual sentence is parsed into a disjunctive dependency structure and structurally matched using word correspondence infortnation. In the course of struc. tural matching, lexical and syntactic ambignities of monolingual sentences are resolved. Finally, from the matching results, monolingual lexical knowledge and translation patterns are acquired.

So far, we have implemented the following: sentence alignment based-on word correspondence information, word correspondence estimation by cooccurrence-frequency-based methods in Gale and Church (1991) and Kay and Röscheisen (1993), structural matching of parallel sentences (Matsumoto et a.1., 1993), and case frame acquisition of Japanese verbs (Utsuro et al, 1993). In the remainder of this paper, we describe the specifications of sentence alignment and word correspondence estimation in sections 3 and 4 , then report the results of small experiments and evaluate our framework in section 5 . 


\section{Sentence Alignment}

\subsection{Bilingual Sentence Alignment Problem}

In this section, we formally define the problem of bilingual sentence alignment. ${ }^{1}$

Let $S$ be a text of $n$ sentences of a language, and $T$ be a text of $m$ sentences of another language and suppose that $S$ and $T$ are translation of each other:

$$
\begin{aligned}
S & =s_{1}, s_{2}, \ldots, s_{n} \\
T & =t_{1}, t_{2}, \ldots, t_{m}
\end{aligned}
$$

Let $p$ be a pair of minimal corresponding segments in texts $S$ and $T$. Suppose that $p$ consists of $x$ sentences $s_{a-x+1}, \ldots, s_{a}$ in $S$ and $y$ sentences $t_{b-y+1}, \ldots, t_{b}$ in $T$ and is denoted by the following:

$$
p=\langle a, x ; b, y\rangle
$$

Note that $x$ and $y$ could be 0 . In this paper, we call the pair of minimal corresponding segments in bilingual texts a sentence bead. ${ }^{2}$ Then, sentences in bilingual texts of $S$ and $T$ are aligned into a sequence $P$ of sentence beads:

$$
P=p_{1}, p_{2}, \ldots, p_{k}
$$

We put some restriction on possibilities of sentence alignment. We assume that each sentence belongs to only one sentence bead and order constraints must be preserved in sentence alignment. Supposing $p_{i}=$ $\left\langle a_{i}, x_{i} ; b_{i}, y_{i}\right\rangle$, those constraints are expressed in the following:

$$
\begin{aligned}
a_{0}=0 & , \quad b_{0}=0 \\
a_{i}=a_{i-1}+x_{i} & , \quad b_{i}=b_{i-1}+y_{i} \quad(1 \leq i \leq k)
\end{aligned}
$$

Suppose that a scoring function $h$ can be defined for estimating the validity of each sentence bead $p_{i}$. Then, bilingual sentence alignment problem can be defined as an optimization problem that finds a sequence $P$ of sentence beads which optimizes the total score $H$ of the sequence $P$ :

$$
H(P)=H_{h}\left(h\left(p_{1}\right), \ldots, h\left(p_{k}\right)\right)
$$

\subsection{Bilingual Sentence Alignment us- ing Word Correspondence Infor- mation}

In this section, we describe the specification of our sentence alignment method based-on word correspondence information. ${ }^{3}$

\footnotetext{
${ }^{1}$ In this paper, we do not describe paragraph alignment process. For the moment, our paragraph alignment program is not reliable enough and the results of sentence alignment are better without paragraph alignment than with paragraph alignment. Since bilingual texts in our bilingual corpus are not so long, the computational cost of sentence alignment is not serious problem even without paragraph alignment.

${ }^{2}$ The term bead is taken from Brown et al. (1991)

${ }^{3}$ We basically use dictionary-based bilingual sentence alignment method originally reported in Murao (1991). The work in Murao (1991) was done under the supervision of Prof. M. Nagao and Prof. S. Sato (JAIST, East).
}

\subsubsection{Score of Sentence Bead}

Before aligning sentences in bilingual texts, content words are extracted from each sentence (after each sentence is morphologically analyzed if necessary), and word correspondences are found using both bilingual dictionaries and statistical information source for word correspondence. Then, using those word corre spondence information, the score $h$ of a sentence bead $p$ is calculated as follows.

First, supposing $p=\langle a, x ; b, y\rangle$, and let $n_{s}(a, x)$ and $n_{t}(b, y)$ be the numbers of content words in the sequences of sentences $s_{a-x+1}, \ldots, s_{a}$ and $t_{b-y+1}, \ldots, t_{b}$ respectively, and $n_{s t}(p)$ be the number of corresponding word pairs in $p$. Then, the score $h$ of $p$ is defined as the ratio of $n_{s t}(p)$ to the sum of $n_{s}(a, x)$ and $n_{t}(b, y)$ :

$$
h(p)=\frac{n_{s t}(p)}{n_{s}(a, x)+n_{t}(b, y)}
$$

\subsubsection{Dynamic Programming Method}

Let $P_{i}$ be the sequence of sentence beads from the beginning of the bilingual text up to the bead $p_{i}$ :

$$
P_{i}=p_{1}, p_{2}, \ldots, p_{i}
$$

Then, we assume that the score $H\left(P_{i}\right)$ of $P_{i}$ follows the recursion equation below:

$$
H\left(P_{i}\right)=H\left(P_{i-1}\right)+h\left(p_{i}\right)
$$

Let $H_{m}\left(a_{i}, b_{i}\right)$ be the maximum score of aligning a part of $S$ (from the beginning up to the $a_{i}\left(=a_{i-1}+x_{i}\right)$ th sentence) and a part of $T$ (from the beginning up to $b_{i}\left(=b_{i-1}+y_{i}\right)$ - th sentence). Then, Equation 1 is transformed into:

$$
\begin{aligned}
& H_{m}\left(a_{i}, b_{i}\right) \\
& \quad=\max _{x_{i}, y_{i}}\left\{H_{m}\left(a_{i}-x_{i}, b_{i}-y_{i}\right)+h\left(\left\langle a_{i}, x_{i} ; b_{i}, y_{i}\right\rangle\right)\right\}
\end{aligned}
$$

where the initial condition is:

$$
H_{m}\left(a_{0}, b_{0}\right)=H_{m}(0,0)=0
$$

We limit the pair $\left(x_{i}, y_{i}\right)$ of the numbers of sentences in a sentence bead to some probable ones. For the moment, we allow only $1-1,1-2,1-3,1-4,2-2$ as pairs of the numbers of sentences:

$$
\begin{array}{r}
\left(x_{i}, y_{i}\right) \in \quad\{(1,1),(1,2),(2,1),(1,3), \\
(3,1),(1,4),(4,1),(2,2)\}
\end{array}
$$

This optimization problem is solvable as a standard problem in dynamic programming. Dynamic programming is applied to bilingual sentence alignment in most of previous works (Brown et al., 1991; Gale and Church, 1993; Chen, 1993). 


\section{Word Correspondence Esti- mation}

In this section, first we describe estimation functions based-on co-occurrence frequencies. Then, we show how to incorporate word correspondence information available in bilingual dictionaries and to estimate word correspondences not included in bilingual dictionaries. Finally, we describe the threshold function for extracting corresponding word pairs.

\subsection{Estimation Function}

In the following, we assume that sentences in the bilingual text are already aligned.

Let $w_{s}$ and $w_{t}$ be words in the texts $S$ and $T$ re spectively, we define the following frequencies:

$$
\begin{aligned}
\operatorname{freq}\left(w_{s}, w_{t}\right)= & \text { (frequency of } w_{s} \text { and } w_{t} \text { 's } \\
& \text { co-occurring in a sentence bead) } \\
\operatorname{freq}\left(w_{s}\right)= & \text { (frequency of } \left.w_{s}\right) \\
\operatorname{freq}\left(w_{t}\right)= & \text { (frequency of } \left.w_{t}\right) \\
N= & \text { (total number of sentence beads) }
\end{aligned}
$$

Then, estimation functions of Gale's (Gale and Church, 1991) and Kay's (Kay and Röscheisen, 1993) are given as below.

\subsubsection{Gale's Method}

Let $a \sim d$ be as follows:

$$
\begin{aligned}
& a=\operatorname{freq}\left(w_{s}, w_{t}\right) \\
& b=\operatorname{freq}\left(w_{s}\right)-\operatorname{freq}\left(w_{s}, w_{t}\right) \\
& c=\operatorname{freq}\left(w_{t}\right)-\operatorname{freq}\left(w_{s}, w_{t}\right) \\
& d=N-a-b-c
\end{aligned}
$$

Then, the validity of word correspondence $w_{s}$ and $w_{t}$ is estimated by the following value:

$$
\begin{aligned}
& h_{g}\left(w_{s}, w_{t}\right) \\
& =\frac{(a d-b c)^{2}}{(a+b)(a+c)(b+d)(c+d)} \\
& =\frac{(a d-b c)^{2}}{\operatorname{freq}\left(w_{s}\right) f r e q\left(w_{t}\right)\left(N-f r e q\left(w_{s}\right)\right)\left(N-f r e q\left(w_{t}\right)\right)}
\end{aligned}
$$

\subsubsection{Kay's Method}

The validity of word correspondence $w_{s}$ and $w_{t}$ is estimated by the following value:

$$
h_{k}\left(w_{s}, w_{t}\right)=\frac{2 f r e q\left(w_{s}, w_{t}\right)}{f r e q\left(w_{s}\right)+f r e q\left(w_{t}\right)} .
$$

\subsection{Incorporating Bilingual Dictio- nary}

By incorporating word correspondence information available in bilingual dictionaries, it becomes easier to estimate word correspondences not included in bilingual dictionaries.

Let $w_{s}$ be a word in the text $S$ and $w_{t}, w_{t}^{\prime}$ be words in the text $T$. Suppose that the correspondence of $w_{s}$ and $w_{t}$ is included in bilingual dictionaries, while the correspondence of $w_{s}$ and $w_{t}^{\prime}$ is not included. Then the problem is to estimate the validity of word correspondence of $w_{s}$ and $w_{t}^{\prime}$.

L,et $f r e q\left(w_{s}, w_{t}\right), \quad f r e q\left(w_{s}, w_{t}^{\prime}\right), \quad f r e q\left(w_{s}\right)$, freq $\left(w_{t}\right)$, and freq $\left(w_{t}^{\prime}\right)$ be the same as above, and freq $\left(w_{s}, w_{t}, w_{t}^{\prime}\right)$ be the frequency of $w_{s}, w_{t}$, and $w_{t}^{\prime}$ 's co-occurring in a sentence bead. Then, we solve the problem above by defining $f r e q^{\prime}\left(w_{s}, w_{t}^{\prime}\right)$, freq $\left(w_{s}\right)$, freq $\left(w_{t}^{\prime}\right)$, and $N^{\prime}$ which become the inputs to Gale's method or Kay's method. We describe two different ways of defining those values.

\section{Estimation I}

One is to estimate all the word correspondences equally except that the co-occurrence of $w_{s}$ and $w_{t}$ is preferred to that of $w_{s}$ and $w_{t}^{\prime}$. freq $\left(w_{s}, w_{t}^{\prime}\right)$, freq $q^{\prime}\left(w_{s}\right)$, freq $\left(w_{t}^{\prime}\right)$, and $N^{\prime}$ are given below: ${ }^{4}$

$$
\begin{aligned}
\operatorname{freq}\left(w_{s}, w_{t}^{\prime}\right) & = \\
\operatorname{freq}\left(w_{s},\right. & \left.w_{t}^{\prime}\right)-\sum_{w_{t}} \operatorname{freq}\left(w_{s}, w_{t}, w_{t}^{\prime}\right) \\
\operatorname{freq}\left(w_{s}\right) & =\operatorname{freq}\left(w_{s}\right) \\
\operatorname{freq}\left(w_{t}^{\prime}\right) & =\operatorname{freq}\left(w_{t}^{\prime}\right) \\
N^{\prime} & =N \\
\left(\operatorname{freq}\left(w_{s}, w_{t}\right)\right. & \left.=\operatorname{freq}\left(w_{s}, w_{t}\right)\right)
\end{aligned}
$$

When $w_{s}, w_{t}$, and $w_{t}^{\prime}$ are co-occurring in a sentence bead, the co-occurrence of $w_{s}$ and $w_{t}$ is preferred and that of $w_{s}$ and $w_{t}^{\prime}$ is ignored. 'Thus, freq' $\left(w_{s}, w_{t}^{\prime}\right)$ is obtained by subtracting the frequency of all those cases from the real co-occurrence frequency of $w_{\mathrm{s}}$ and $w_{t}^{\prime}$. But, freq $\left(w_{s}\right)$ and $f r e q^{\prime}\left(w_{t}^{\prime}\right)$ are the same as the real frequencies and the estimated word correspondences reflect the real co-occurrence frequencies in the input text. (Compare with Estimation II.) Word correspondences both included and not included in bilingual dictionaries are equally estimated their validities.

\section{Estimation II}

The other is to remove from the input text all the co-occurrences of word pairs included in bilingual dictionaries. freq $\left(w_{s}, w_{t}^{\prime}\right), f r e q^{\prime}\left(w_{s}\right), f r e q^{\prime}\left(w_{t}^{\prime}\right)$, and $N^{\prime}$ are given below:

\footnotetext{
${ }^{4}$ It can happen that, within a sentence bead, one word of a language has more than one corresponding words of the opposite language and all the correspondences are included in bilingunl dictionaries. In that case, formalizations in this section need some modifications.
} 


$$
\begin{array}{r}
\operatorname{freq}^{\prime}\left(w_{s}, w_{t}^{\prime}\right)= \\
\operatorname{freq}\left(w_{s}, w_{t}^{\prime}\right)-\sum_{w_{t}} \operatorname{freq}\left(w_{s}, w_{t}, w_{t}^{\prime}\right) \\
\operatorname{freq}\left(w_{s}\right)=\operatorname{freq}\left(w_{s}\right)-\sum_{w_{t}} \operatorname{freq}\left(w_{s}, w_{t}\right) \\
\operatorname{freq}\left(w_{t}^{\prime}\right)=\operatorname{freq}\left(w_{t}^{\prime}\right)-\sum_{w_{s}^{\prime}} \operatorname{freq}\left(w_{s}^{\prime}, w_{t}^{\prime}\right)
\end{array}
$$

(the correspondence of $w_{s}^{\prime}$ and $w_{t}^{\prime}$ is included in bilingual dictionaries)

$$
N^{\prime}=N
$$

With this option, after all the co-occurrences of word pairs included in bilingual dictionaries are removed from the input text, word correspondences not included in bilingual dictionaries are estimated their validities.

In the following sections, we temporarily adopt Estimation I for estimating word correspondences not included in bilingual dictionaries. It is necessary to further investigate and compare the two estimation methods with large-scale experiments.

\subsection{Threshold Function}

As a threshold function for extracting appropriate corresponding word pairs, we use a hyperbolic function of word frequency and estimated value for word correspondence.

At first, we define the following variables and constants: ${ }^{5}$

$$
\begin{aligned}
& x=\text { (co-occurrence frequency) } \\
& y=\text { (estimated value for word correspondence) } \\
& a=\text { (constant for eliminating low frequency } \\
& \text { words) ( } 1.0 \text { for both } h_{g} \text { and } h_{k} \text { ) } \\
& b=\text { (constant for eliminating words) } \\
& \text { with low estimated value) } \\
& \text { ( } 0.1 \text { for } h_{g} \text { and } 0.3 \text { for } h_{k} \text { ) } \\
& c=\text { (lower bound of word frequency) } \\
& \text { ( } 2.5 \text { for both } h_{g} \text { and } h_{k} \text { ) }
\end{aligned}
$$

Then, the threshold function $g(x, y)$ is defined as below:

$$
g(x, y)=\frac{x(y-b)}{a} \quad(x>c)
$$

And the condition for extracting corresponding word pairs is given below:

$$
g(x, y)>1 \quad, \quad x>c
$$

When using extracted word correspondences in sentence alignment and structural matching, at present we ignore the estimated values and use estimated word correspondences and word correspondences in bilingual dictionaries equally.

\footnotetext{
${ }^{5}$ Note that values for constants are determined temporarily and need further investigation with large-scale experiments. Especially, constants related to word frequency have to be tuned to the length of texts.
}

\section{$5 \quad$ Experiment and Evaluation}

In this section, we report the results of a small experiment on aligning sentences in bilingual texts and statistically estimating word correspondences.

The sentence alignment program and the word correspondence estimation program are named AlignCO. The processing steps of AlignCO are as follows:

1. Given a bilingual text, content words are extracted from each sentence.

2. A Japanese-English dictionary of about 50,000 entries is consulted and word correspondence information is extracted for content words of each sentence.

3. The sentence alignment program named Align $C O / A$ aligns sentences in the input text by the method stated in section 3.2 .

4. Given the aligned sentences in the bilingual text, the word correspondence estimation program named Align $C O / C$ estimates word correspondences which are not included in the JapaneseEnglish dictionary with option Estimation $\mathbf{I}$ in section 4.2 .

5. Combining word correspondence information available in the Japanese- English dictionary and estimated by $\operatorname{Align} C O / C$, sentences in the input text are realigned.

As input Japanese-Linglish bilingual texts, we use two short texts of different length - 1) "The Dilemma of National Development and Democracy" (305 Japanese sentences and 300 English sentences, henceforth "dilemma"), 2) "Pacific Asia in the PostCold-War World" (134 Japanese sentences and 123 English sentences, henceforth "cold-war"). Since the results of Gale's method and Kay's method did not differ so much, we show the result of Gale's method only.

\subsection{Sentence Alignment}

The followings are five best results of sentence alignment before and after estimating word correspondences not included in the Japanese-English dictionary. The results are improved after estimating word correspondences not included in the bilingual dictionary.

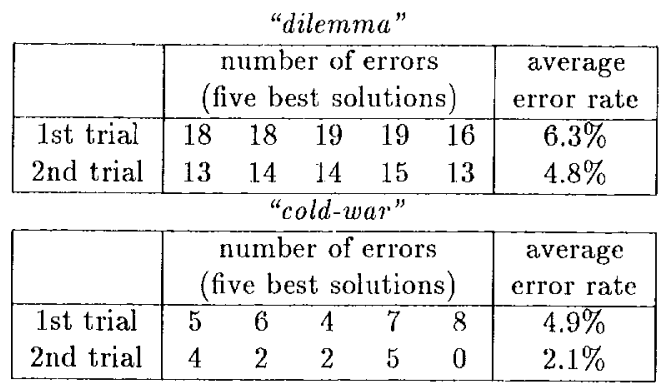




\subsection{Word Correspondence Estimation}

We classify the estimated word correspondences into three categories, "correct", "part of phrase", and "wrong". "part of phrase" means that the estimated word correspondence can be considered as part of corresponding phrases. "error rate" is the ratio of the number of "wrong" worl correspondences to the total number.

\begin{tabular}{|c|c|c|c|c|}
\hline \multicolumn{5}{|c|}{ "dilemmu" } \\
\hline total & correct & $\begin{array}{l}\text { part of } \\
\text { phrase }\end{array}$ & wrong & crror rate \\
\hline 87 & 53 & 30 & 4 & $4.6 \%$ \\
\hline \multicolumn{5}{|c|}{ "cold-war" } \\
\hline total & correct & $\begin{array}{l}\text { part of } \\
\text { phrase }\end{array}$ & wrong & error rate \\
\hline 37 & 19 & $\overline{10}$ & 8 & $21.6 \%$ \\
\hline
\end{tabular}

The result of "dilemma" is better than that of "cold war". This is because the former is longer than the latter.

The followings are example word correspondences of each category where $f_{s}, f_{t}$, and $f_{s t}$ are $f r e q\left(w_{s}\right)$, freq $\left(w_{t}\right)$, and $\operatorname{freq}\left(w_{s}, w_{t}\right)$ respectively. The parenthesized correspondence is not extracted by the threshold function.

\begin{tabular}{|c|c|c|c|c|c|}
\hline \multicolumn{6}{|c|}{ correct } \\
\hline$w_{s}$ & $w_{t}$ & $h_{g}$ & $f_{s}$ & $f_{t}$ & $f_{s t}$ \\
\hline 天ルタン & sultan & 0.75 & 4 & 3 & 3 \\
\hline 战椺 & press & 0.80 & 5 & 4 & 4 \\
\hline llill & liberal & 0.64 & 20 & 15 & 14 \\
\hline 綾激 & economic & 0.32 & 33 & 19 & 15 \\
\hline \multicolumn{6}{|c|}{ part of phrase } \\
\hline$w_{s}$ & $w_{t}$ & $h_{g}$ & $f_{s}$ & $f_{t}$ & $\bar{f}_{s t}$ \\
\hline 女 & civilian & 1.00 & 6 & 6 & 6 \\
\hline 文 & supremacy & 0.83 & 6 & 5 & 5 \\
\hline 1 & civilian & 0.69 & 6 & 6 & 5 \\
\hline $1 x^{\prime}$ & supremacy & 0.83 & 6 & 5 & 5 \\
\hline 渗少 & supremacy & 0.44 & 4 & 5 & 3 \\
\hline (㒄分分 & civilian & 0.37 & 4 & 6 & 3) \\
\hline \multicolumn{6}{|c|}{ wrong } \\
\hline$w_{s}$ & $w_{t}$ & $\bar{h}_{g}$ & $f_{s}$ & $\overline{f_{t}}$ & $f_{s t}$ \\
\hline 急林 & does & 0.49 & 6 & 3 & 3 \\
\hline A波淮 & and & 0.41 & 47 & 62 & 5 \\
\hline
\end{tabular}

Most of "correct" correspondences are proper names like "Хルタン - sultan", or those which have different parts of speech, like "lilll (nomn) - lib eral (adjective)" and "終济 (noun) - economic (adjective)", or those which can be considered as translation equivalents but not included in the Japanese-Lnglish

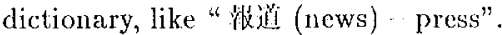

The examples of "part of phrase" form a phrase

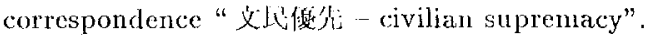

The former "wrong" correspondence "密沬 (meaning) - does" comes from the correspondence of long

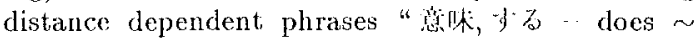

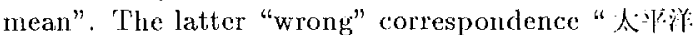
(pacific ocean) - and" is extracted by Gale's method because both freq(太湔) and freq(and) are high and close to the total number of sentence beads. This correspondence is not extracted by Kay's method.
Then, in Fig. 2, we illustrate the relation between the estimated value $h_{g}\left(w_{s}, w_{t}\right)$ of Gale's method and the co-occurrence frequency freq $\left(w_{s}, w_{t}\right)$ for the text "dilemma". The threshold function seems optimized so that it extracts as many word correspondences of the category "correct" and "part of phrase" as possible, and extracts as few word correspondences of the category "wrong" as possible.

\section{Concluding Remarks}

This paper described a unified framework for bilingual text matching by combining existing hand written bilingual dictionaries and statistical techniques. Hspecially, we described a method for aligning sentences using word correspondence information, and a method for estimating word correspondences not included in bilingual dictionaries.

Estimated word correspondence information will improve the results of structural matching of bilingual sentences. It will be reported in the future. With the same techniques as those for estimating word colrespondences, it is quite easy to estimate correspondences of phrases such as noun phrases and idiomatic expressions. 'Then, the results of structural matching will be much more improved.

In order to improve the accuracy of sentence alignment, we need to combine our word-correspondencebased inethod with those length-based methods in Brown ot al. (1991) and Gale and Church (1993). In the case of Japanese-Finglish texts, the wordbased method in Brown et al. (1991) might be better than the character-based method in Gale and Church (1993).

\section{References}

Brown, P. F., Cocke, J., Pietra, S. A., Pietra, V. J. D. et al. (1990). A statistical approach to machine translation, Computational Linguistics 16(2): $79-85$.

Brown, P. F., Lai, J. C. and Mercer, R. L. (1991). Aligning sentences in bilingual corpora, Proceedings of the 29th Annual Meeting of ACL, pp. $169-176$.

Brown, P. F., Pietra, S. A. D., Pietra, V. J. D. and Mercer, R. L. (1993). The mathematics of statistical machine translation: Parameter estimation, Computational Linguistics 19(2): 263-311.

Chen, S. F. (1993). Aligning sentences in bilingual corpora using lexical information, Proceedings of the 31th Annual Meeting of ACL, pp. 9-16.

Church, K. W. (1993). Char_align: A program for aligning parallel texts at the character level, Proceedings of the 31th Annual Meeting of ACL, pp. 1-8.

Dagan, I., Itai, A. and Schwall, U. (1991). 'T'wo languages are more informative than one, Pro- 

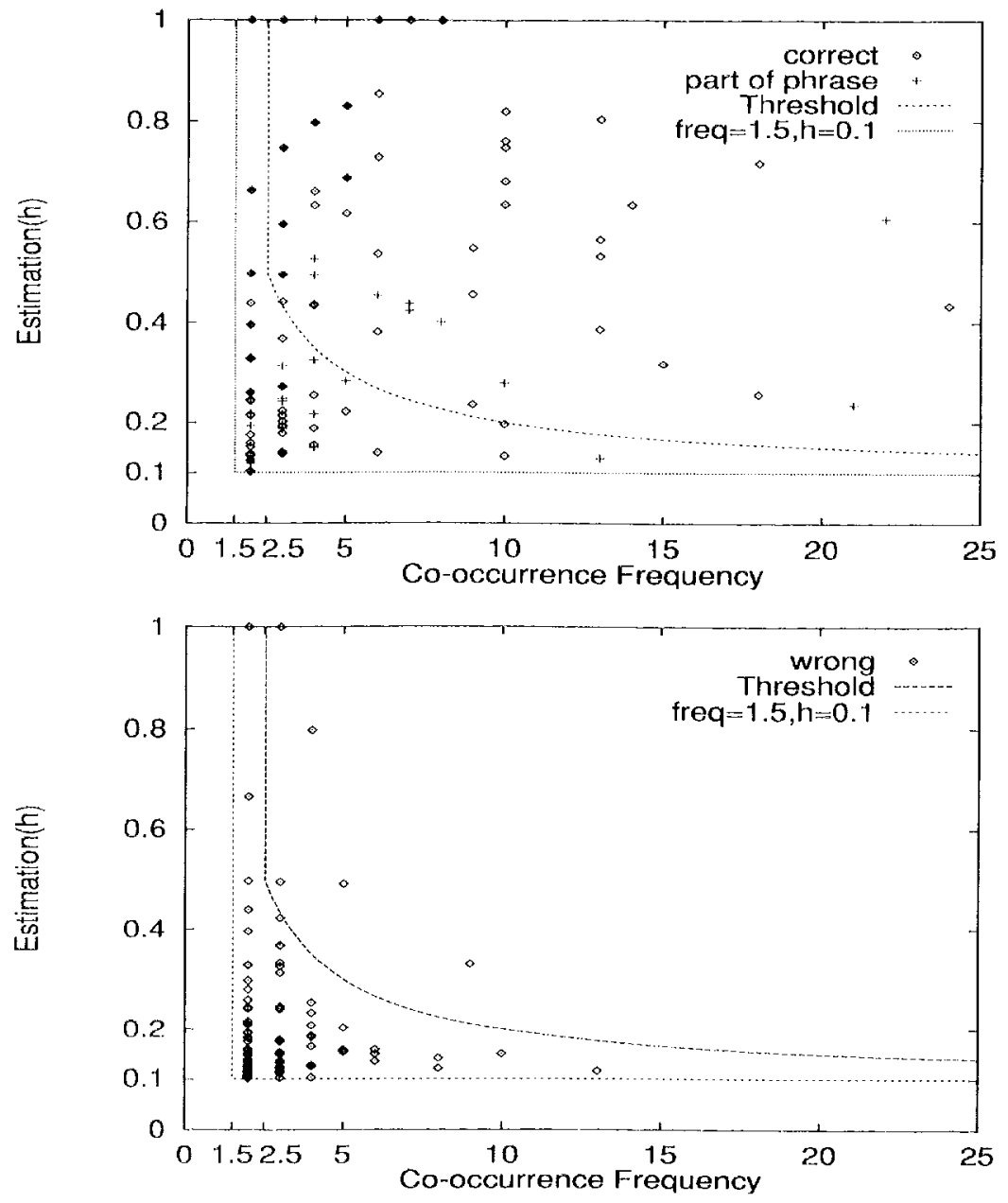

fig. 2: Wstimation per Co-occurrence Frequency of Word Correspondences ( dilemma")

ceedings of the 29th Annual Meeting of ACL, pp. $130-137$.

Gale, W. A. and Church, K.W. (1993). A program for aligning sentences in bilingual corpora, Computational Linguistics 19(1): $75 \cdot 102$.

Gale, W. and Church, K. (1991). Identifying word correspondences in parallel texts, Proceedings of the 4th DARPA Speech and Natural Language Workshop, pp. 152--157.

Kaji, H., Kida, Y. and Morimoto, Y. (1992). Learning translation templates from bilingual text, Procedings of the 14th COLING, pp. 672-678.

Kay, M. and Röscheisen, M. (1993). Texttranslation alignment, Computational Linguistics 19(1): 121--142.

Klavans, J. and Tzoukermann, E. (1990). The BICORD System: Combining lexical information from bilingual corpora and machine readable dictionaries, Proceedings of the 13th COLING, Vol. 3, pp. 174-179.

Kupiec, J. (1993). An algorithm for finding noun phrase correspondences in bilingual corpora,
Proceedings of the 31th Annual Meeting of ACL, pp. $17 \sim 22$.

Matsumoto, Y., Ishimoto, II. and Utsuro, T. (1993). Structural matching of bilingual texts, proceed- $_{\text {- }}$ ings of the 31th Annual Meeting of $A C L$, pp. 2330.

Murao, H. (1991). Studies on bilingual text align ment, Bachelor Thesis, Kyoto University. (in Japanese).

Sadler, V. and Vendelmans, R. (1990). Pilot implementation of a bilingual knowledge bank, Proceedings of the 13th COLING, Vol. 3, pp. 449451.

Utsuro, T., Matsumoto, Y. and Nagao, M. (1992). Lexical knowledge acquisition from bilingual corpora, Proceedings of the 14th COLING, pp. 581587.

Utsuro, T., Matsumoto, Y. and Nagao, M. (1993). Verbal case frame acquisition from bilingual corpora, Proceedings of the 13th IJCAI, pp. 11501156. 